\title{
Socioeconomic disparities in obesity among children and future actions to fight obesity in China
}

\author{
Hyunjung Lim $^{1,2}$, Youfa Wang ${ }^{3,4}$ \\ ${ }^{1}$ Department of Medical Nutrition, Graduate School of East-West Medical Science, Kyung Hee University, Yongin, Korea; ${ }^{2}$ Research Institute of \\ Medical Nutrition, Kyung Hee University, Seoul, Korea; ${ }^{3}$ Fisher Institute of Health and Well-being, Department of Nutrition and Health Sciences, \\ College of Health, Ball State University, Muncie, IN, USA; ${ }^{4}$ Global Health Institute, School of Public Health, Xi'an Jiaotong University, Xi'an \\ 710000 , China \\ Correspondence to: Hyunjung Lim. Department of Medical Nutrition, Graduate School of East-West Medical Science, Kyung Hee University, 1732 \\ Deogyeong-daero, Giheung-gu, Yongin-si, Gyeonggi-do 17104, Republic of Korea. Email: hjlim@khu.ac.kr; Youfa Wang. Fisher Institute of Health \\ and Well-being, Department of Nutrition and Health Sciences, College of Health, Ball State University, Muncie, IN 47306, USA. \\ Email: ywang26@bsu.edu; youfawang@gmail.com. \\ Provenance: This is an invited article commissioned by the Guest Section Editor Dr. Ying Zhao, MM (Department of Laboratory Medicine, the First \\ Affiliated Hospital, Zhejiang University School of Medicine, Hangzhou, China). \\ Comment on: Dong Y, Jan C, Ma Y, et al. Economic development and the nutritional status of Chinese school-aged children and adolescents from \\ 1995 to 2014: an analysis of five successive national surveys. Lancet Diabetes Endocrinol 2019;7:288-99.
}

Submitted Dec 09, 2019. Accepted for publication Dec 22, 2019.

doi: 10.21037/atm.2019.12.134

View this article at: http://dx.doi.org/10.21037/atm.2019.12.134

\section{Previous and new studies: the similar alarming messages}

As the second largest economy and the most populous country in the world, China has experienced many dramatic changes in social and economic development in the last 30 years. There have been many changes in the lives and lifestyles of Chinese people, while there are many remarked shifts in their eating and physical activity behaviors, including consuming more energy dense foods and having increasingly sedentary lifestyle. As a result, the prevalence of overweight and obesity among Chinese has been increasing rapidly over the last two or three decades (1-3). On the other hand, the prevalence of undernutrition has dropped dramatically. These have been happening among children and adults $(1,2,4)$. National data showed that in recent years approximately $43 \%$ of adults and $20 \%$ of children in China are overweight or obese $(2,4,5)$.

Using data collected from the large nationally representative surveys conducted during 1995 and 2014, the recent study published by Dong et al. (2) reported the changing patterns of nutritional status among Chinese children aged 7-18 years old, and examined the association between nutritional status and socioeconomic indicators.
The nutritional status outcomes they studied included stunting, thinness, overweight, and obesity. They examined a set of socioeconomic indicators including gross domestic product (GDP) per capita, Engel coefficient (the proportion of household income spent on food), and urbanisation ratio. In addition, the differences between urban and rural areas were investigated. The data covered a large number of children $(\mathrm{N}=1,054,602)$ included in the Chinese National Survey on Students' Constitution and Health (CNSSCH) from 29 provinces in China. The concept and contents of this study are very important and have serve to provide valuable information.

Overall, the patterns reported in this study are consistent with findings from some previous studies around the world (6-8), including some from China and our work (1). This study confirmed that with the social economic development in China, the rates of stunting (from $8.1 \%$ to $2.4 \%$ ) and thinness (from $8.1 \%$ to $2.4 \%$ ) had decreased, and that of overweight and obesity had increased (from $5.3 \%$ to $20.5 \%$ ) during 1995-2014 (2). Findings from the study have many important public health implications to help guide future programs and policies in China. A very interesting finding is that over time, the nutritional status of urban-rural gap gradually decreased and the association 
between socioeconomic status (SES) and overweight and obesity became stronger in rural- than in urban areas (2). Another related important finding from the study is that compared to their urban counterparts, rural children still had more nutritional problems (2). They should receive more attention and support in the future, in particular, in government supported programs and in school-based intervention programs.

The study by Dong and colleagues has some limitations. First, it did not report results for minority populations in China. Second, it used cross-sectional survey data and thus could not test causal relationships. Third, the SES measures were not individual-level or household-level ones, but were aggregated population level measures. The study has many strengths. It studied a set of nutritional status outcomes, SES indicators, and their associations, and used national data collected from a large number of children over two decades. In addition, it used vigorous analysis approaches and provided comprehensive results.

\section{Children and adults: trends in obesity and chronic diseases in China}

Childhood obesity has become a health concern of high interest worldwide including in China. The prevalence of childhood overweight and obesity has increased steadily in China $(9,10)$. Our team's 2017 study based on a longitudinal dataset from a nationwide study in China showed some regional differences in obesity, and the prevalence of childhood obesity, including overweight, increased from $11.7 \%$ in 1991 to $25.2 \%$ in 2011 (9). The prevalence in boys was higher (by about 1.5-2 times) and had increased faster than in girls.

The long-term health consequences of childhood obesity will appear in adulthood as it increases the risks of many non-communicable chronic diseases (NCDs). It is likely that China will pay a high price in the years to come for today's high rates of overweight and obesity in children. Previous studies have shown that the prevalence of overweight and obesity increased in all age, sex, and residence regions in China. As China's obesity rate has increased, obesityand diet-related NCDs such as high blood pressure, cardiovascular disease (CVD), and type 2 diabetes have increased in the last 20 years, becoming the most important cause of death (1). According to a China national report in 2015, i.e., the Nutrition and Health Status (11), they reported an alarming increase in obesity and NCDs over the past decade with improvement in nutritional status and reduced undernutrition problems among Chinese including children. Obesity prevalence, NCDs morbidity and mortality had increased steadily since 2002 . Moreover, the prevalence of hypertension (18.8\% to $25.2 \%)$ and diabetes (2.6\% to $9.7 \%$ ) had increased between 2002 and 2012. The main NCDs (mainly CVDs, cancer and chronic respiratory diseases) were the leading causes of deaths, accounting for $79.4 \%$ of total mortality in 2012 (4).

There are emerging evidences indicate that cardiovascular health outcomes (such as body mass index, total cholesterol, blood pressure, and glucose) and their influencing behaviors (smoking, physical activity, and diet) have worsened in recent years in China even among children. For example, one of our studies conducted in Beijing during 2004-2014 showed that only $19.5 \%$ of boys and $22.0 \%$ of girls had ideal cardiovascular health in 2004 . The figures became much worse in 2014 (boys: 9.8\%; girls: $16.0 \%)(12)$. This likely would predict future increased morbidity and mortality from CVDs when these young people become adults. Effective intervention programs that promote healthy lifestyles are urgently needed in China, and some need to target at children.

\section{China and the world: large disparities in the prevalence of childhood obesity worldwide and within countries and the association between SES and obesity}

What has been happening in China is also a reflection of the worldwide situation. There are large disparities in the prevalence of childhood obesity worldwide and within countries. Childhood obesity prevalence is still much higher in Western and industrialized countries than most developing countries. The prevalence in many developing countries especially in low-income countries in Africa and Southeast Asia is still very low, is lower than $10 \%(10,13,14)$. However, the prevalence in some developing countries including China, has increased at a much faster annual rate. At present, the prevalence in some countries or among some groups in some developing countries (e.g., in major cities in China) has reached the same level as in Western countries like the United States $(2,4)$. The Americas and Eastern Mediterranean regions had higher prevalence of childhood overweight and obesity (about 30-40\%) than the European (20-30\%), south-east Asian, western Pacific, and African regions (10-20\% in the latter three) (15).

There has been a longstanding interest to study the relationship between obesity and SES, which has many 
important public health and policies implications. A rich body of literature has shown that SES affects obesity, and the association varies among populations and countries. Several comprehensive reviews have summarized findings from studies from worldwide $(16,17)$. In general, many studies reported that low-SES groups in industrialized countries and high-SES groups in developing countries (including China) are more likely to be overweight or obese than their counterparts.

The obesity-SES relationship is complex as shown by previous studies including ours $(10,18-21)$. It varies by factors such as age, sex, ethnicity, and countries; it changes over time; and Obesity and SES are interrelated because obesity can negatively affect people's SES by limiting educational and employment opportunities $(18,19)$. Our previous research shows that the association between SES and obesity in the United States has become weaker, which was due to the fact that the increase of obesity affected different-SES groups differently (21). Other research has indicated that in some developing countries including China, the direction of the SES-obesity association has changed over time $(14,15,21)$. In the past, the prevalence was higher in higher-SES group, but in recent years, the lower-SES groups had higher prevalence in some developing countries.

According to many studies in developed and developing countries, the SES group, which has more access to energydense foods, is more likely to become obese. Urban children especially in high-SES families are at increased risk for excess weight gain in some developing countries (19). A large study showed that the prevalence of overweight was higher among children from less affluent families (about $80 \%$ of Western countries and $50 \%$ of Central European countries) compared to children from more affluent families (8). In addition, girls from less affluent families were more likely to be overweight, but the opposite was found for boys (8).

\section{Obesity prevention and control in China}

Obesity is very difficult and costly to cure once develops. If people can maintain healthy body weight, it helps prevent many NCDs such as type 2 diabetes and CVDs. Childhood obesity prevention is the key to fight the obesity epidemic. Childhood obesity can be prevented through a healthy lifestyle, including healthy eating and proper physical activity. School-programs are important to help empower children to develop life-long healthy habits and maintain healthy body weight. Future interventions should be tailored to the specific needs of subpopulations (e.g., by their SES), to more efficiently fight the epidemic and reduce health disparities. Obesity intervention efforts need the support and participation of many different sectors of the society, ranging from government agencies to private sectors. Broad coalitions including international collaborations are needed. Often this is challenging, but is possible. Some of the lessons learned in the field of tobacco control are using for obesity intervention, although the latter is much more complex and challenging.

Given the fast-growing rates of obesity and related NCDs in China, in recent years there are growing interest and support for obesity prevention and control in China. One example is that during 2015-2019, with the support of the Chinese Nutrition Society, our international research team with expertise in diverse fields ranging from nutrition, clinical medicine to exercise science, which was led by Professors Youfa Wang, Mingxiao Sun and Yuexin Yang, worked closely in synthesizing evidences in the field from China and abroad, and published the "China Blue Paper On Obesity Prevention and Control" (in short, "China Obesity Blue Paper") in April 2019, which is in Chinese. The report was developed aiming to provide guidelines and help enhance future obesity and NCD prevention and control efforts in China $(3,5)$. It systematically examined the epidemiology of obesity and obesity prevention and control related policies in China. The report has made a series of recommendations for future research and public health campaigns to fight obesity in China. Some of the recommendations were refined and reported in our recent article published in Lancet Global Health $(3,16,22)$.

Following are some of the recommendations we made for China's future efforts to fight the obesity epidemic $(3,16,22)$ :

(I) Making government responsible and enhancing cross-sector collaboration;

(II) Making better use of health professionals' institutions to promote large-scale, populationlevel educational programs and campaigns;

(III) Engaging families and individuals in obesity prevention and control by making individuals accountable for their health;

(IV) Improving obesogenic environments by establishing and improving regulations and policies on fast food expansion, marketing, food labelling, and nutrition education; establishing and improving regulations and policies on food storage and restaurant and food wholesale surveillance and management; 
encouraging healthy food supply and choices in the workplace; improving the school environment; improving built environments that encourage physical activity;

(V) Improving obesity treatment by standardizing clinical guidelines, practice, and services; enhancing professional training in obesity diagnosis, prevention, and treatment; and promoting selfmanagement of overweight and obese patients;

(VI) Reforming health insurance policies to facilitate obesity prevention and treatment.

In conclusion, obesity has become a serious public health threat in China, which affects both adults and children, although large disparities in the rates exist within China across populations and regions. The development of national policies and population-based public health programs specifically designed to target at obesity are needed to combat the obesity and NCDs epidemic and to promote public health in China. In order to fight the obesity problem in China, strong government commitment and appropriate investment, extensive participation and cooperation in various social and health sectors are important. This will help achieve the national goals of "Healthy China 2030".

\section{Acknowledgments}

None.

\section{Footnote}

Conflicts of Interest: The authors have no conflicts of interest to declare.

Ethical Statement: The authors are accountable for all aspects of the work in ensuring that questions related to the accuracy or integrity of any part of the work are appropriately investigated and resolved.

\section{References}

1. Wang $\mathrm{Y}, \mathrm{Mi}$ J, Shan $\mathrm{XY}$, et al. Is China facing an obesity epidemic and the consequences? The trends in obesity and chronic disease in China. Int J Obes (Lond) 2007;31:177-88.

2. Dong Y, Jan C, Ma Y, et al. Economic development and the nutritional status of Chinese school-aged children and adolescents from 1995 to 2014: an analysis of five successive national surveys. Lancet Diabetes Endocrinol 2019;7:288-99.

3. Wang YF, Sun MX, Yang YX. China blue paper on obesity prevention and control (In Chinese). Peking University Medical Publisher, 2019.

4. Wang Y, Wang L, Qu W. New national data show alarming increase in obesity and noncommunicable chronic diseases in China. Eur J Clin Nutr 2017;71:149-50.

5. Wang YF, Sun MX, Xue H, et al. Understanding the China Blue Paper on Obesity Prevention and Control and policy implications and recommendations for obesity prevention and control in China. Zhonghua Yu Fang Yi Xue Za Zhi 2019;53:875-84.

6. Zhang Q, Wang Y. Using concentration index to study changes in socio-economic inequality of overweight among US adolescents between 1971 and 2002. Int J Epidemiol 2007;36:916-25.

7. Wang Y, Lobstein T. Worldwide trends in childhood overweight and obesity. Int J Pediatr Obes 2006;1:11-25.

8. Due P, Damsgaard MT, Rasmussen M, et al.

Socioeconomic position, macroeconomic environment and overweight among adolescents in 35 countries. Int $\mathrm{J}$ Obes (Lond) 2009;33:1084-93.

9. Jia P, Xue H, Zhang J, et al. Time Trend and Demographic and Geographic Disparities in Childhood Obesity Prevalence in China-Evidence from Twenty Years of Longitudinal Data. Int J Environ Res Public Health 2017. doi: 10.3390/ijerph14040369.

10. Wang Y, Monteiro C, Popkin BM. Trends of obesity and underweight in older children and adolescents in the United States, Brazil, China, and Russia. Am J Clin Nutr 2002;75:971-7.

11. The Chinese National Health and Family Planning Commission. [Internet]. 2015. Available online: http:// mp.weixin.qq.com/s?_biz=MjM5OTIyODc1NA==\&mid $=205782183 \& \mathrm{idx}=1 \& \mathrm{sn}=12 \mathrm{~d} 59495 \mathrm{ff} 4866 \mathrm{ffa} 0 \mathrm{~d} 92023 \mathrm{e} 4 \mathrm{efc}$ 40e\&scene $=1 \#$ rd

12. Dong H, Yan Y, Liu J, et al. Alarming trends in ideal cardiovascular health among children and adolescents in Beijing, China, 2004 to 2014. Int J Cardiol 2017;231:264-70.

13. NCD Risk Factor Collaboration (NCD-RisC). Worldwide trends in body-mass index, underweight, overweight, and obesity from 1975 to 2016: a pooled analysis of 2416 population-based measurement studies in 128.9 million children, adolescents, and adults. Lancet 2017;390:2627-42.

14. Jones-Smith JC, Gordon-Larsen P, Siddiqi A, et al. 
Cross-national comparisons of time trends in overweight inequality by socioeconomic status among women using repeated cross-sectional surveys from 37 developing countries, 1989-2007. Am J Epidemiol 2011;173:667-75.

15. Wang $Y$, Lim $H$. The global childhood obesity epidemic and the association between socio-economic status and childhood obesity. Int Rev Psychiatry 2012;24:176-88.

16. Jia P, Xue H, Cheng X, et al. Association of neighborhood built environments with childhood obesity: Evidence from a 9-year longitudinal, nationally representative survey in the US. Environ Int 2019;128:158-64.

17. Bush NR, Allison AL, Miller AL, et al. Socioeconomic Disparities in Childhood Obesity Risk: Association With an Oxytocin Receptor Polymorphism. JAMA Pediatr 2017;171:61-7.

Cite this article as: Lim H, Wang Y. Socioeconomic disparities in obesity among children and future actions to fight obesity in China. Ann Transl Med 2019;7(Suppl 8):S377. doi: 10.21037/ atm.2019.12.134
18. McLaren L. Socioeconomic status and obesity. Epidemiol Rev 2007;29:29-48.

19. Sobal J, Stunkard AJ. Socioeconomic status and obesity: a review of the literature. Psychol Bull 1989;105:260-75.

20. Wang Y. Cross-national comparison of childhood obesity: the epidemic and the relationship between obesity and socioeconomic status. Int J Epidemiol 2001;30:1129-36.

21. Wang Y, Zhang Q. Are American children and adolescents of low socioeconomic status at increased risk of obesity? Changes in the association between overweight and family income between 1971 and 2002. Am J Clin Nutr 2006;84:707-16.

22. Wang Y, Xue H, Sun M, et al. Prevention and control of obesity in China. Lancet Glob Health 2019;7:e1166-7. 